\title{
Identification of an Immune Gene Expression Signature for Predicting Lung Squamous Cell Carcinoma Prognosis
}

\author{
Yubo Yan, ${ }^{1}$ Minghui Zhang, ${ }^{2}$ Shanqi $X{ }^{2}$, and Shidong $X u \mathbb{D}^{1}$ \\ ${ }^{1}$ Department of Thoracic Surgery, Harbin Medical University Cancer Hospital, Harbin, China \\ ${ }^{2}$ Department of Medical Oncology, Harbin Medical University Cancer Hospital, Harbin, China \\ Correspondence should be addressed to Shidong Xu; xsdhrb@163.com
}

Received 9 March 2020; Revised 2 June 2020; Accepted 11 June 2020; Published 28 June 2020

Academic Editor: Khac-Minh Thai

Copyright (c) 2020 Yubo Yan et al. This is an open access article distributed under the Creative Commons Attribution License, which permits unrestricted use, distribution, and reproduction in any medium, provided the original work is properly cited.

\begin{abstract}
Growing evidence indicates that immune-related biomarkers play an important role in tumor processes. This study investigates immune-related gene expression and its prognostic value in lung squamous cell carcinoma (LUSC). A cohort of 493 samples of patients with LUSC was collected and analyzed from data generated by the TCGA Research Network and ImmPort database. The $\mathrm{R}$ coxph package was employed to mine significant immune-related genes using univariate analysis. Lasso and stepwise regression analyses were used to construct the LUSC prognosis prediction model, and clusterProfiler was used for gene functional annotation and enrichment analysis. The Kaplan-Meier analysis and ROC were used to evaluate the model efficiency in predicting and classifying LUSC case prognoses. We identified 14 immune-related genes to incorporate into our prognosis model. The patients were divided into two subgroups (Risk-H and Risk-L) according to their risk score values. Compared to Risk-L patients, Risk-H patients showed significantly improved overall survival (OS) in both training and testing sets. Functional annotation indicated that the 14 identified genes were mainly enriched in several immune-related pathways. Our results also revealed that a risk score value was correlated with various signaling pathways, such as the JAK-STA signaling pathway. Establishment of a nomogram for clinical application demonstrated that our immune-related model exhibited good predictive prognostic performance. Our predictive prognosis model based on immune signatures has potential clinical implications for assessing the overall survival and precise treatment for patients with LUSC.
\end{abstract}

\section{Introduction}

Lung cancer remains the leading cause of cancer incidence and mortality worldwide [1]. Non-small cell lung cancer (NSCLC) is the most common type of lung cancer and is classified into two major histological subtypes, lung adenocarcinoma (LUAD) and lung squamous cell carcinoma (LUSC), each with distinct genomic and immunological profiles [2]. The discovery of epidermal growth factor receptor (EGFR), anaplastic lymphoma kinase (ALK), and ROS proto-oncogene 1 (ROS1) gene targets and the development of corresponding target drugs have prolonged the survival of patients with NSCLC [3]. Currently, progress has been slow in the development of LUSC treatments due to the lack of effective targets; however, continuous developments in immunotherapy have provided a new direction for LUSC treatment [4]. Immunocyte infiltration, which is speculated to represent the active tumor response, can be detected among most solid tumors in humans; specifically, lymphocyte infiltration in LUSC has certain survival benefits [5]. Therefore, understanding the immune gene signatures of LUSC is highly significant as it could have predictive prognosis implications.

At present, the tumor-node-metastasis (TNM) classification system has been recognized as the most meaningful indicator for prognosis and can inform therapeutic decisions for LUAD as well as LUSC treatment [6]. Nonetheless, this classification system is imprecise because various progression levels and overall survival (OS) results can be observed among cases in the same stage. Therefore, novel markers are urgently needed to recognize patients with high recurrence risk. A precisely indicated prognosis significantly 
affects a clinician's decision to recommend adjuvant therapy. Additionally, there is increasing need to improve prognosis prediction tools.

Biomarkers can reliably predict disease prognosis as well as patient survival. As a result, they are meaningful in the decision-making process for clinical LUSC treatment. In recent years, an increasing number of articles have recommended that gene expression profiles can be applied to predict and stratify the survival prognosis of LUSC cases $[7,8]$. However, the role of immune-related genes in LUSC is unclear. Therefore, openly accessible large databases that contain gene expression profiles allow us to mine creditable biomarkers for predicting and classifying LUSC prognosis.

This study aimed at establishing and verifying a prognosis prediction model for LUSC based on genes related to immunity and patient clinical features derived from the Cancer Genome Atlas (TCGA) Research Network and ImmPort database.

\section{Materials and Methods}

2.1. Data Collection. Gene expression and clinical LUSC patient data were downloaded from the TCGA Research Network (https://www.cancer.gov/tcga), and the gene set related to immunity was obtained from the ImmPort database (https://www.immport.org). The raw data were preprocessed as follows: (1) samples without clinical data were removed; (2) normal tissue sample data were removed; (3) genes with fragments per kilobase per million reads (FPKM) values of 0 in more than half the samples were removed; and (4) the expression profiles of immune-related genes were saved. After preprocessing, 493 samples comprising 1421 immune-related genes were utilized for further model analysis. The 493 samples were randomized into training and test sets. All samples underwent 500 iterations of random grouping with replacement to eliminate the impact of random allocation bias on model stability. Data in the training $(n=245)$ and test $(n=248)$ sets are presented in Table 1. There was no statistically significant difference between the two sets, which indicated reasonable sample grouping.

2.2. Prognostic Signature. The correlation of immune-related gene expression with patient OS was assessed through the univariate Cox proportional hazards regression analysis using the survival coxph function of the $\mathrm{R}$ package. Genes with $p$ values $<0.05$ were identified as candidate genes. Subsequently, the number of candidate genes was reduced according to the least absolute shrinkage and selection operator lasso-Cox method using the glmnet and MASS function of the R package. Genes most significantly related to immunity were selected to construct the prognosis risk score model. The risk score model was formulated as follows:

$$
\text { Risk score }=\sum_{i=0}^{n} \beta i \times X i \text {, }
$$

where $\beta i$ represents the coefficient of every gene and $\chi i$ stands for gene expression level (FPKM). The median risk score value was the threshold for classifying samples into
TABLE 1: Patient characteristics with lung squamous cell carcinoma in training and testing sets.

\begin{tabular}{|c|c|c|c|c|}
\hline Clinical features & Overall & Training set & Testing set & $p$ value \\
\hline OS & 493 & 245 & 248 & 0.9383 \\
\hline Event & 493 & 245 & 248 & 0.9293 \\
\hline Alive & 284 & 140 & 144 & \\
\hline Dead & 209 & 105 & 104 & \\
\hline $\mathrm{T}$ & 493 & 245 & 248 & 0.4717 \\
\hline $\mathrm{T} 1$ & 114 & 49 & 65 & \\
\hline $\mathrm{T} 2$ & 286 & 146 & 140 & \\
\hline $\mathrm{T} 3$ & 70 & 40 & 30 & \\
\hline $\mathrm{T} 4$ & 23 & 10 & 13 & \\
\hline $\mathrm{N}$ & 493 & 242 & 246 & 0.7437 \\
\hline N0 & 316 & 160 & 156 & \\
\hline N1 & 127 & 62 & 65 & \\
\hline $\mathrm{N} 2$ & 40 & 19 & 21 & \\
\hline N3 & 5 & 1 & 4 & \\
\hline NX & 5 & 3 & 2 & \\
\hline M & 493 & 208 & 204 & 0.6093 \\
\hline M0 & 405 & 206 & 199 & \\
\hline M1 & 7 & 2 & 5 & \\
\hline MX & 81 & 37 & 44 & \\
\hline Stage & 493 & 244 & 245 & 0.4364 \\
\hline I & 241 & 116 & 125 & \\
\hline II & 158 & 88 & 70 & \\
\hline III & 83 & 38 & 45 & \\
\hline IV & 7 & 2 & 5 & \\
\hline $\mathrm{X}$ & 4 & 1 & 3 & \\
\hline Age & 493 & 245 & 248 & 0.6387 \\
\hline $0 \sim 50$ & 22 & 9 & 13 & \\
\hline $50 \sim 60$ & 73 & 40 & 33 & \\
\hline $60 \sim 70$ & 181 & 85 & 96 & \\
\hline $70 \sim 80$ & 191 & 97 & 94 & \\
\hline $80 \sim 100$ & 26 & 14 & 12 & \\
\hline Subdivision & 493 & 235 & 240 & 0.8473 \\
\hline Bronchial & 10 & 5 & 5 & \\
\hline L-Lower & 74 & 33 & 41 & \\
\hline L-Upper & 135 & 68 & 67 & \\
\hline R-Lower & 106 & 52 & 54 & \\
\hline R-Middle & 18 & 10 & 8 & \\
\hline R-Upper & 132 & 67 & 65 & \\
\hline Gender & 493 & 245 & 248 & 0.9648 \\
\hline Female & 128 & 63 & 65 & \\
\hline Male & 365 & 182 & 183 & \\
\hline
\end{tabular}

high-risk (Risk-H) or low-risk (Risk-L) groups. ROC and the Kaplan-Meier (KM) analyses were carried out to evaluate model efficiency, stability, and accuracy in predicting and classifying LUSC case prognoses.

2.3. Functional Annotations. Eventually, 14 genes were selected and their gene families annotated according to 


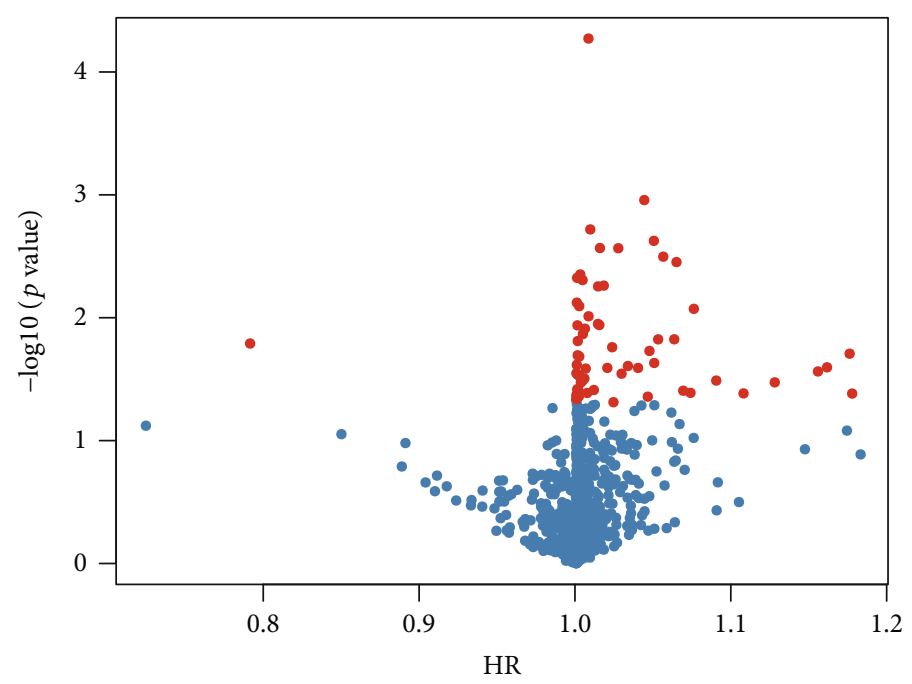

(a)

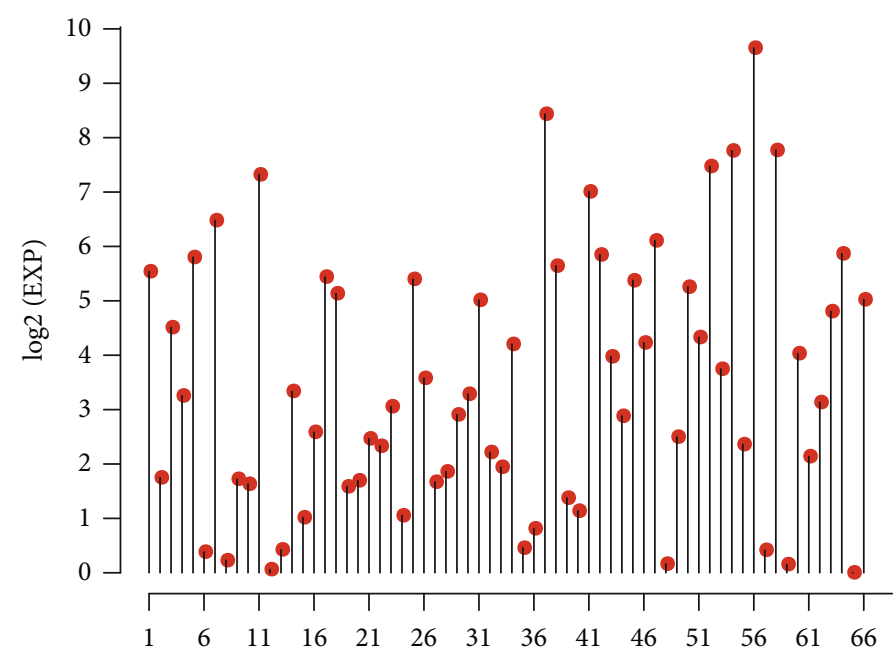

(b)

FIgure 1: Differential gene expression in lung squamous cell carcinoma. (a) The relationships of the -log10 ( $p$ values) and HR. (b) The expression levels of 66 differentially expressed genes. Red dots represent significantly different immune-related genes $(p<0.05)$ regarding prognosis.

human gene classification within the HUGO Gene Nomenclature (HGNC) database. The $\mathrm{R}$ package clusterProfiler was employed to carry out enrichment analysis on the 14 screened genes related to immunity and specific to prognosis. The KEGG enrichment analysis score was evaluated using the ssGSEA function of the R package GSVA [9]. Association with the risk score value was also calculated. Clustering analysis was then carried out according to the pathway enrichment score for each sample.

\subsection{Association between Risk Score Value and Clinical} Features. Associations between relevant clinical factors (such as stage ( $T, N$, or $M)$, subdivision, age, and smoking habit) and risk score value were analyzed. Then, a nomogram model was constructed, and a forest plot was drawn according to relevant clinical features and risk score values. The associations between risk score value and clinical features related to patient survival were also analyzed.
2.5. Statistical Analyses. Independent subgroups were analyzed using the Chi-square test or Fisher's exact test. Univariate and multivariate analyses were performed using the Cox regression. Differences in OS between high- and low-risk groups were evaluated according to the Kaplan-Meier survival curve. The sensitivity and specificity of the diagnosis and prognosis prediction model were determined and assessed using the ROC area under the curve (AUC). The Kruskal-Wallis test was used to evaluate the relationships of risk score with different clinical factors. A two-tailed $p$ value of $<0.05$ was recognized as statistically significant. Statistical analyses were performed using the R software (Version 3.5.5; R Core Team, 2016).

\section{Results}

3.1. Data Processing. Sixty-six immune-related, prognosisspecific genes were mined. The $p$ value relationships of the 


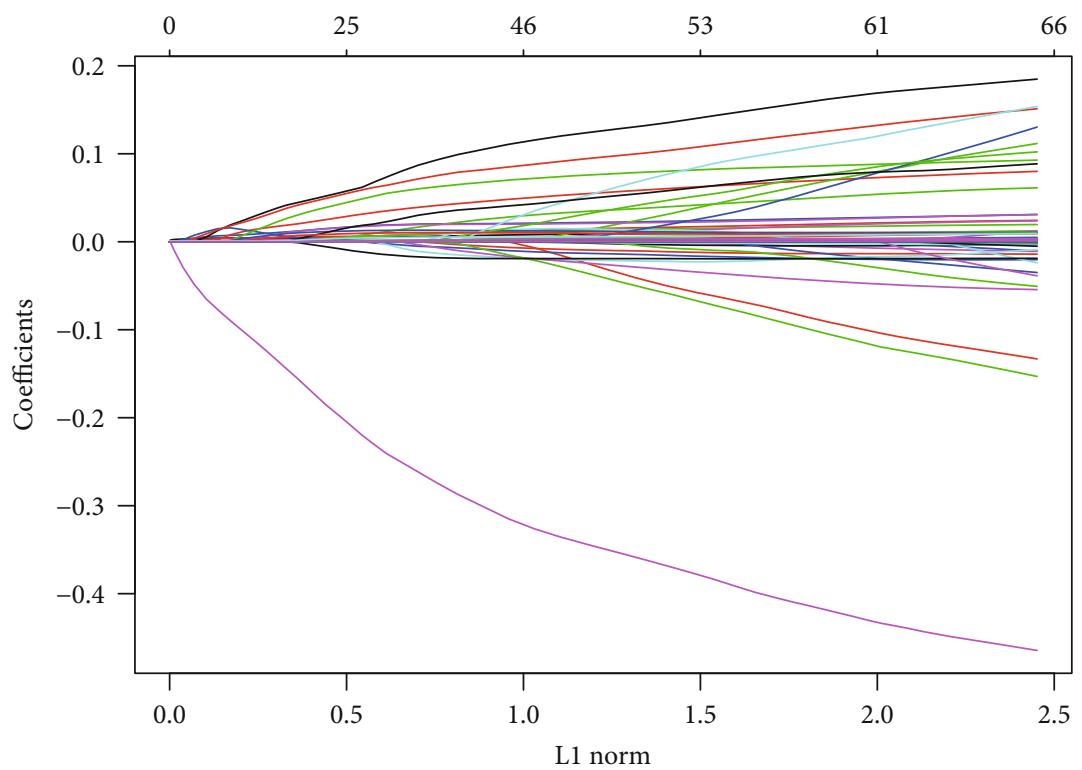

(a)

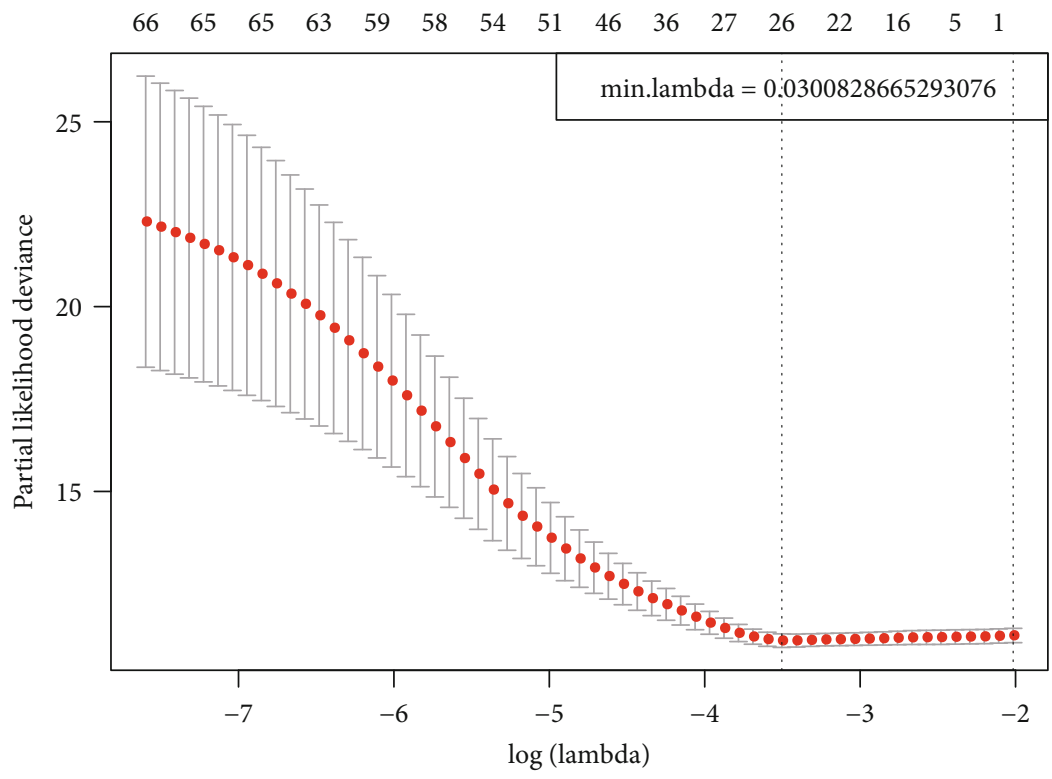

(b)

FIGURE 2: Construction of the prognosis prediction model for LUSC patients by LASSO. (a) The changing trajectory of each independent variable. The horizontal axis represents the log value of the independent variable lambda, and the vertical axis represents the coefficient of the independent variable. (b) Confidence intervals for each lambda.

66 genes with hazard ratios (HRs) and expression levels are displayed in Figure 1.

3.2. Establishment of the Prognosis Prediction Model. Sixtysix immune-related genes were identified, although that number was inappropriately high for use in clinical detection. Therefore, the scope of genes related to immunity was narrowed to maintain high accuracy. The 66 genes were compressed through lasso regression to reduce the number of genes incorporated in the risk model. The variation trajectories for all independent variables (Figure 2(a)) suggested that the coefficients of a larger number of independent parameters were close to 0 as lambda gradually increased. The con- fidence interval (CI) under every lambda (Figure 2(b)) revealed that the best model was obtained at a lambda value of 0.03 , which was consequently chosen for the eventual model that included 26 immunity-related genes. In addition, the MASS of the R package was utilized in stepwise regression analysis based on Akaike data criteria to obtain 14 genes used to construct the risk model.

Each sample from the training cohort was then incorporated into the formula for calculating the risk score value. The OS for all samples is shown in Figure S1. Analysis of the model efficiency in predicting the 1-5-year OS resulted in a mean AUC value reaching 0.703 (Figure 3(a)). Sample distributions in Risk-H and Risk-L groups under different 


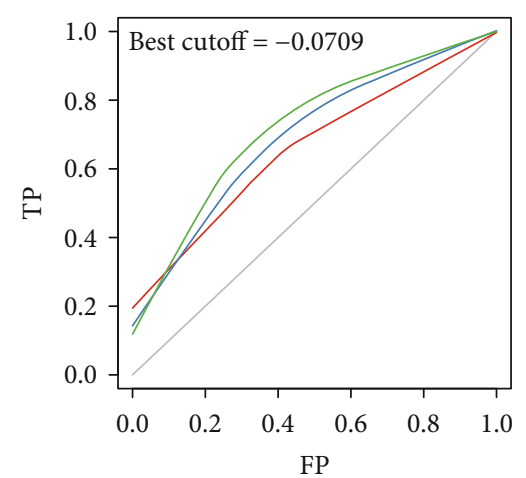

-1 years $(\mathrm{AUC}=0.675)$
-3 years $(\mathrm{AUC}=0.706)$
5 years $(\mathrm{AUC}=0.736)$

(a)

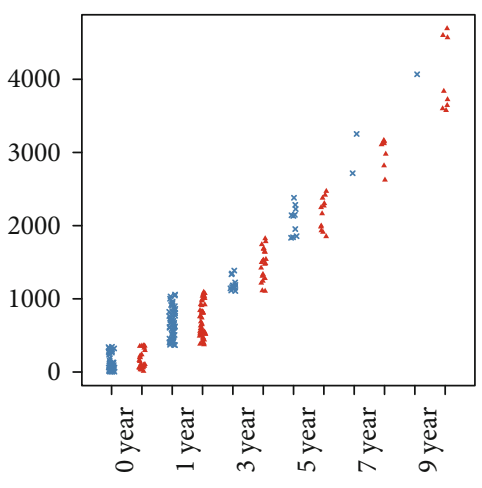

$\times$ Risk-H

$\triangle$ Risk-L

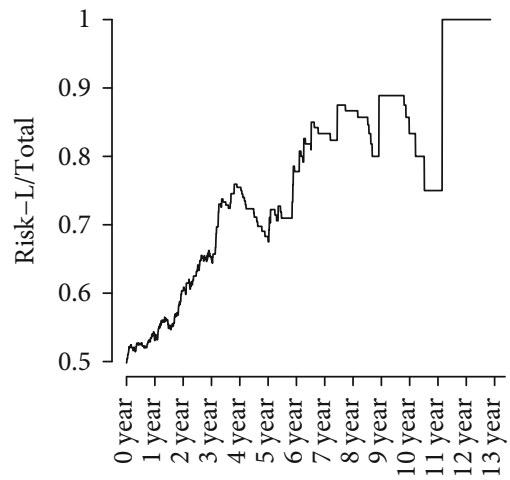

(b)

(c)

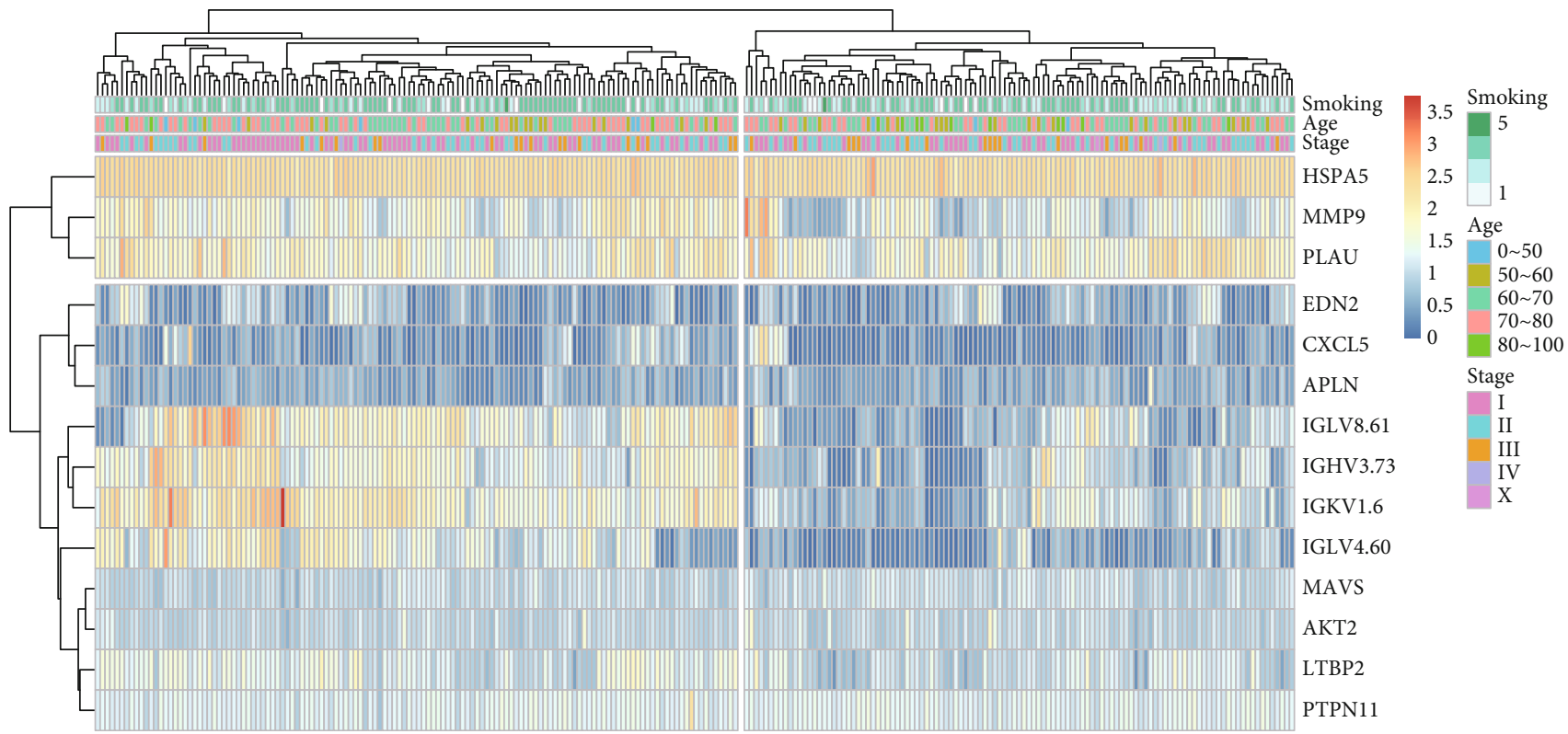

(d)

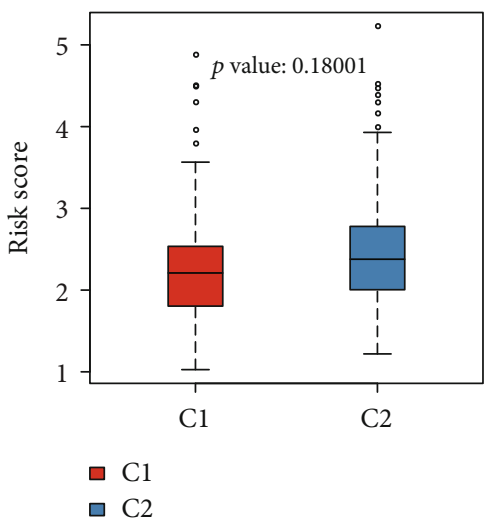

(e)

FIGURE 3: Verification of the stability of the prognosis prediction model for patients with lung squamous cell carcinoma in the training cohort. (a) Survival predicted ROC curves for the training cohort. (b) Distribution of samples in Risk-H and Risk-L groups of the training cohort divided by different OS. (c) The proportion of low-risk samples in total samples varies with OS. (d) Clustering results of the training cohort. (e) Differences in risk score values between Risk-H and Risk-L groups clustered by gene expression in the training cohort. 


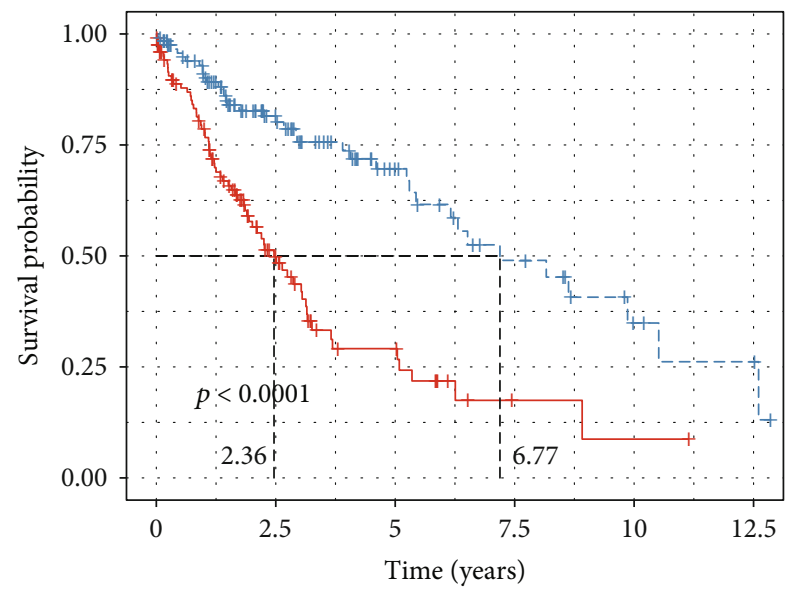

Number at risk

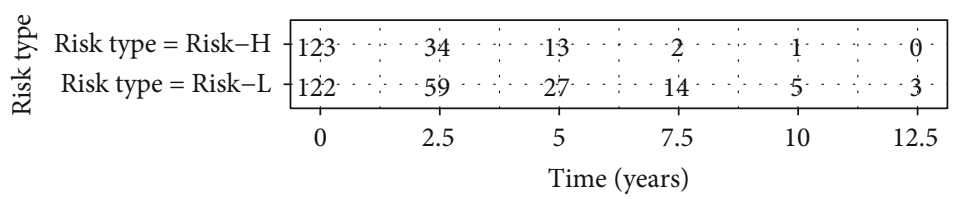

Risk type

+ Risk type $=$ Risk $-\mathrm{H}$

-+ - Risk type $=$ Risk-L

(a)

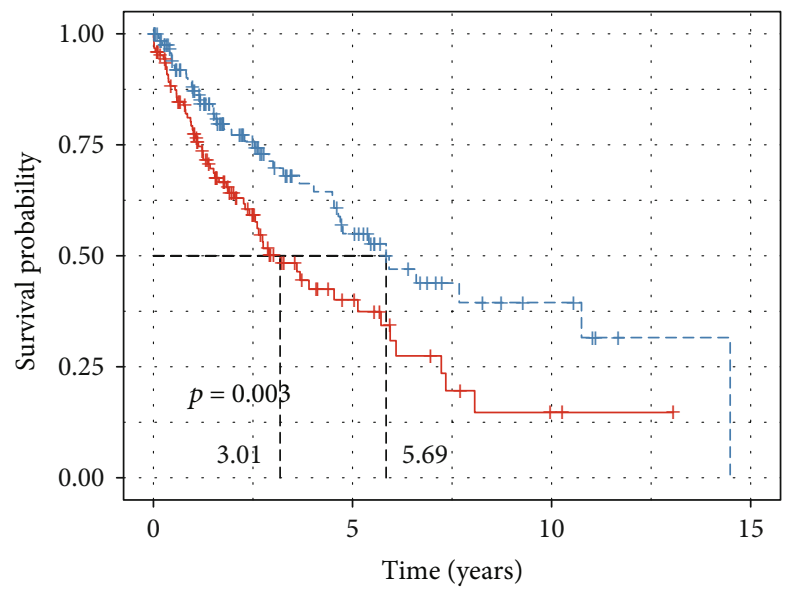

Number at risk

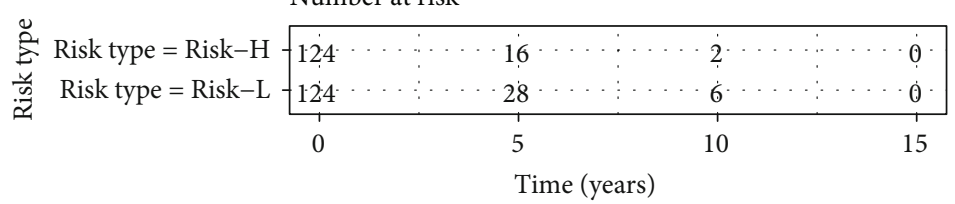

Risk type

+ Risk type $=$ Risk $-\mathrm{H}$

-+- Risk type $=$ Risk $-\mathrm{L}$

(b)

Figure 4: Continued. 


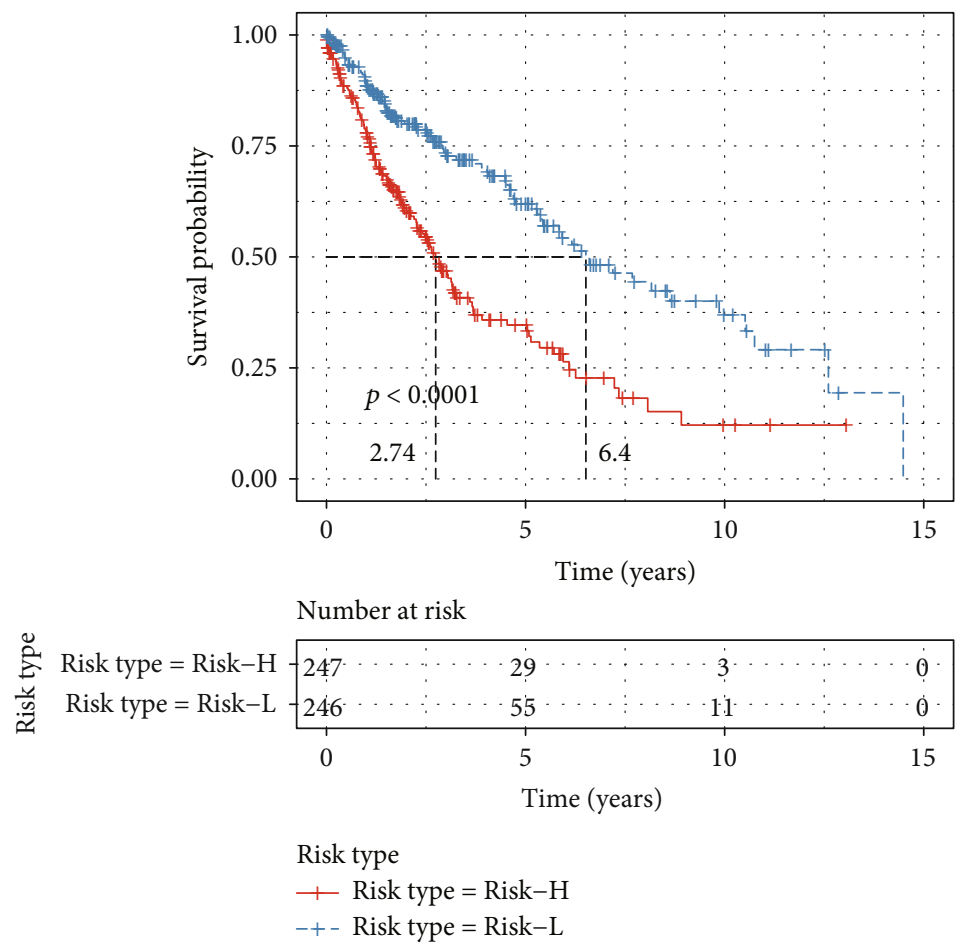

(c)

Figure 4: The Kaplan-Meier survival curve of the 14-gene risk model in predicting the Risk-H and Risk-L groups on the training set (a), testing set (b), and all samples (c).

TABLE 2: Gene function annotation results.

\begin{tabular}{lcc}
\hline Gene family & Genes & $p$ value \\
\hline Endogenous ligands & EDN2/CXCL5/APLN & 0.0003 \\
Latent transforming growth factor beta-binding proteins & LTBP2 & 0.0030 \\
Heat shock $70 \mathrm{kDa}$ proteins & HSPA5 & 0.0108 \\
M10 matrix metallopeptidases & MMP9 \\
Caspase recruitment domain containing & MAVS \\
SH2 domain containing & PTPN11 \\
Pleckstrin homology domain containing & AKT2 \\
Unknown & IGLV8.61/IGHV3.73/IGLV4.60/PLAU/IGKV1.6: \\
\hline
\end{tabular}

OS durations suggested that the 5-year sample size of the Risk-H group was reduced relative to that of Risk-L group (Figures 3(b) and 3(c)). The sample clustering results in the training cohort are presented in Figure 3(d). The 14 genes were clustered into high and low expression groups (Figure $3(\mathrm{e})$ ). To verify the credibility of the prognosis prediction model, the expression profiles of the 14 genes were collected from the test cohort and incorporated into the verification model. The risk score values for the samples in the test cohort corresponded with those in the training cohort (Figure S2). To further verify model creditability and stability in prognosis prediction, the expression profiles of the 14 genes collected from 493 samples were incorporated into the model to calculate the risk score values. The results were consistent with the test set validation results (Figure S3). Taken together, the prognosis prediction model based on 14 immune-related gene expression profiles displayed superb stability and predictive accuracy in identifying immunerelated characteristics.

KM survival curves were plotted for the risk model based on 14 genes in the Risk-H and Risk-L groups of the training cohort, test cohort, and the whole dataset (combined cohort). The KM survival curves of the training, test, and combined cohorts are displayed in Figure 4(a) $(p<0.001)$, Figure 4(b) $(p=0.003)$, and Figure $4(c)(p<0.001)$, respectively.

3.3. Functional Annotation of Immunity-Related Genes. The 14 gene families annotated based on human gene classification in the HGNC database (Table 2) were enriched in the endogenous ligands and latent transforming growth factor $\beta$-binding proteins (LTBP) gene families. Moreover, the expression levels of four genes (END2, CXCL5, APLN, and $L T B P 2$ ) from these two gene families differed significantly between the Risk-H and Risk-L groups (Figure 5). 


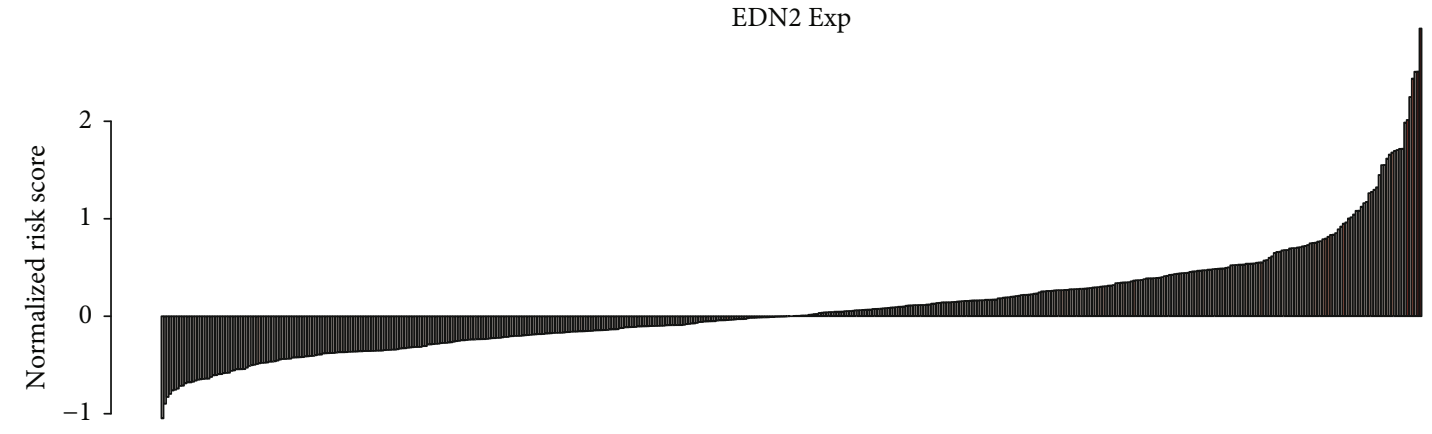

Samples

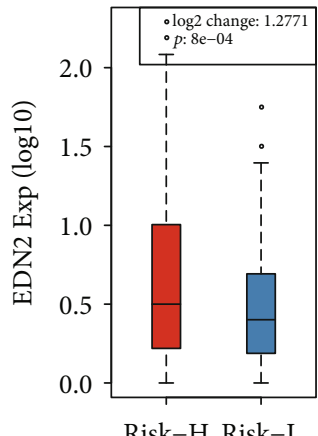

Risk-H Risk-L

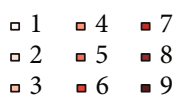

(a)

CXCL5 Exp

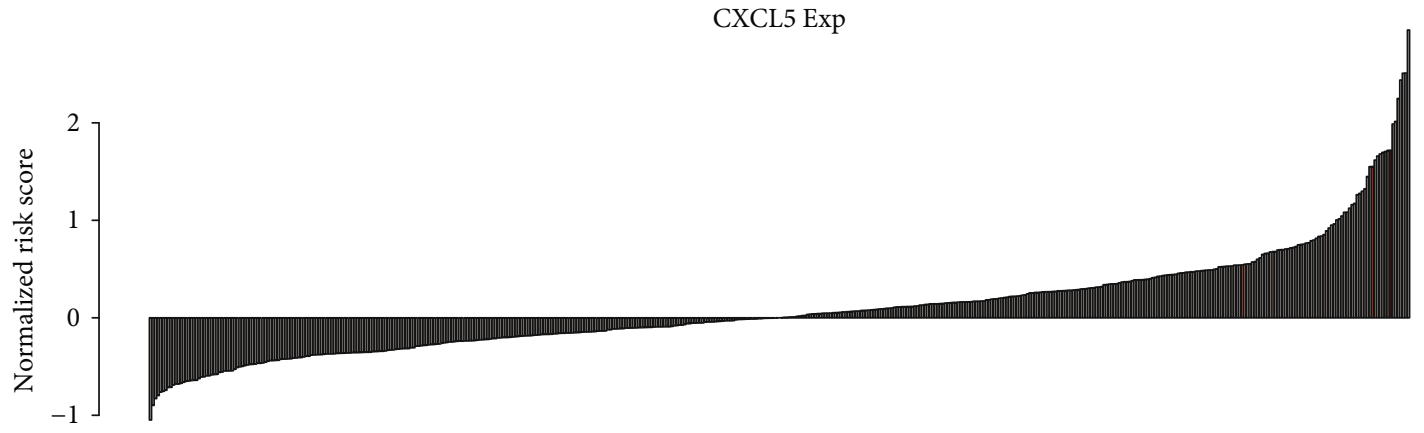

Samples

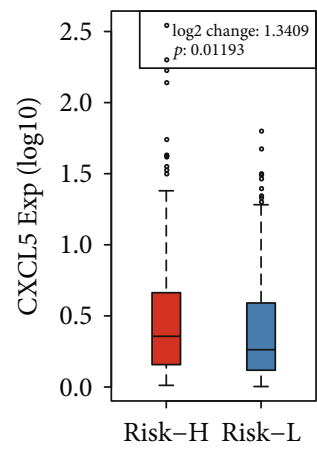

$\square 1 \quad \square \quad \square 7$

$\begin{array}{lll}-1 & -5 & -8\end{array}$

- $3 \quad 6 \quad-9$

(b)

APLN Exp
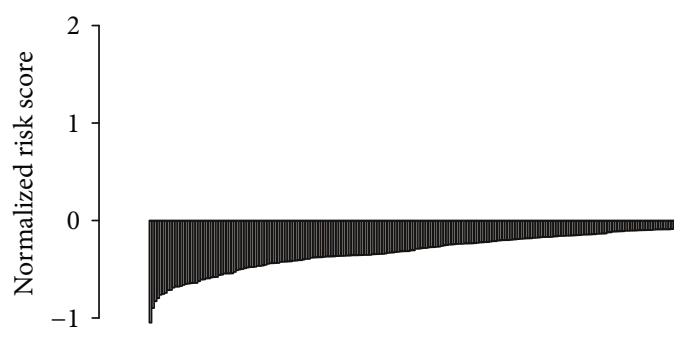

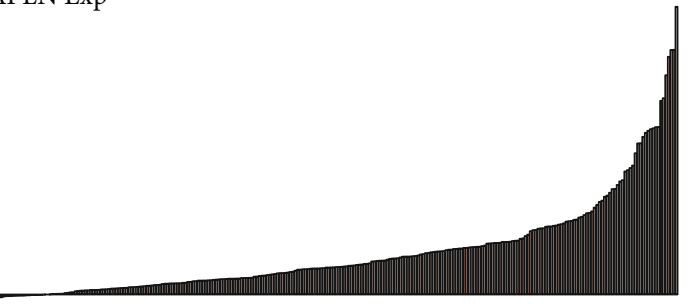

Samples

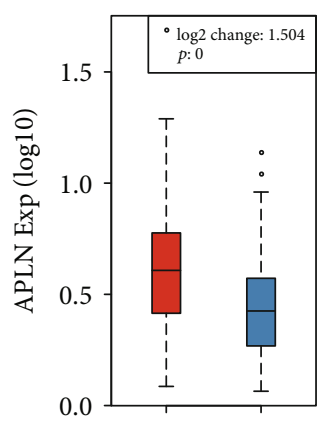

Risk-H Risk-L

$\begin{array}{lll}\square 1 & \square & -7 \\ \square 2 & \square & -8 \\ \square 3 & \square 6 & -9\end{array}$

(c)

Figure 5: Continued. 


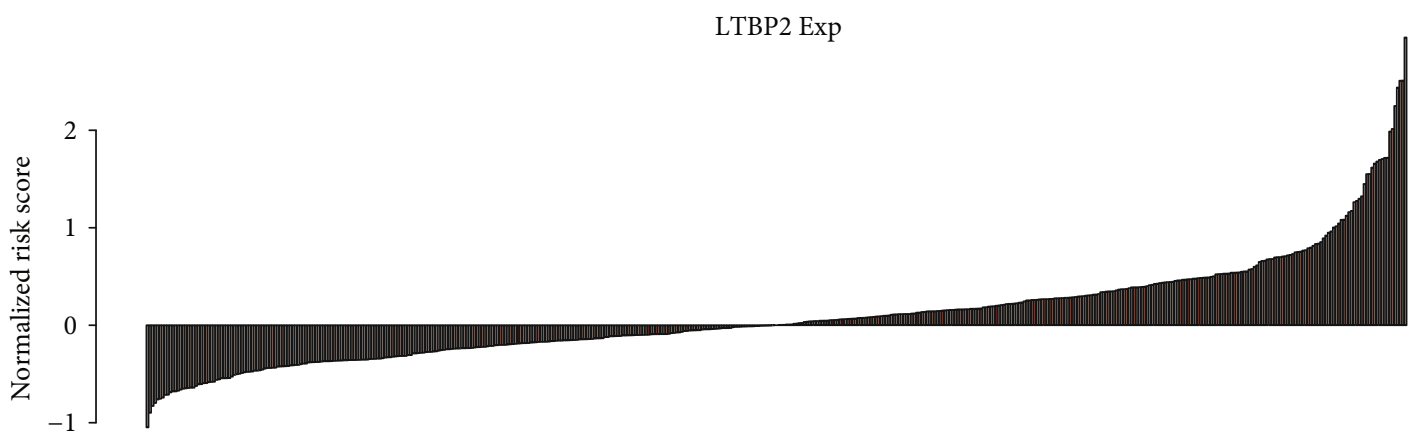

Samples

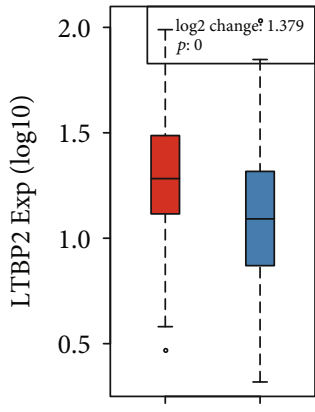

Risk-H Risk-L
$\square$ 口 $4 \quad \square$
- $2 \quad 5 \quad-8$
- $3 \quad 6 \quad-9$

(d)

Figure 5: The expression differences of the EDN2 (a), CXCL5 (b), APLN (c), and LTBP2 (d) between the Risk-H and Risk-L groups.

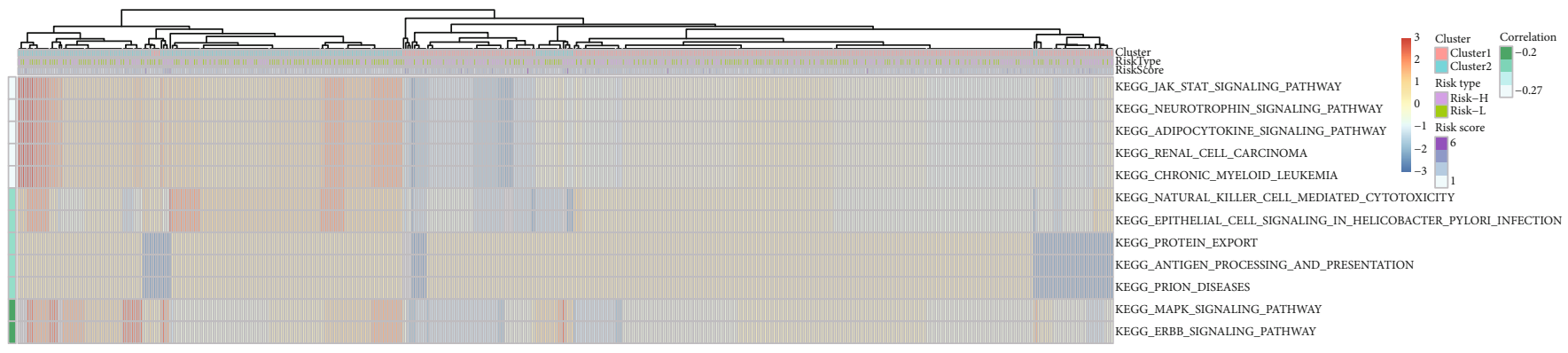

FIgURE 6: Correlation of risk score with signaling pathways.

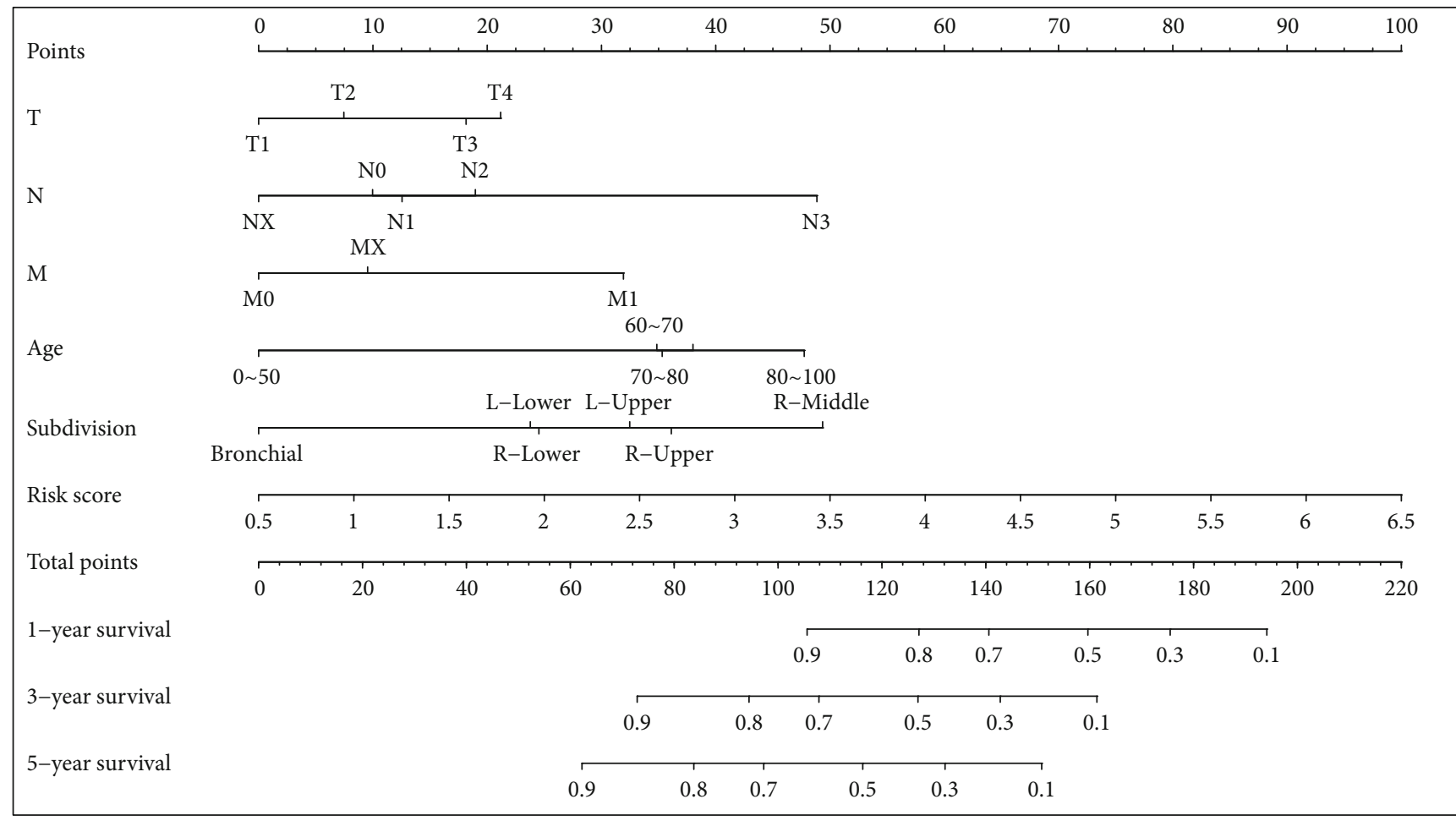

FIgURE 7: The nomogram model constructed by combining the stage- $\mathrm{T}$, stage- $\mathrm{N}$, stage- $\mathrm{M}$, age, subdivision, and risk score. 


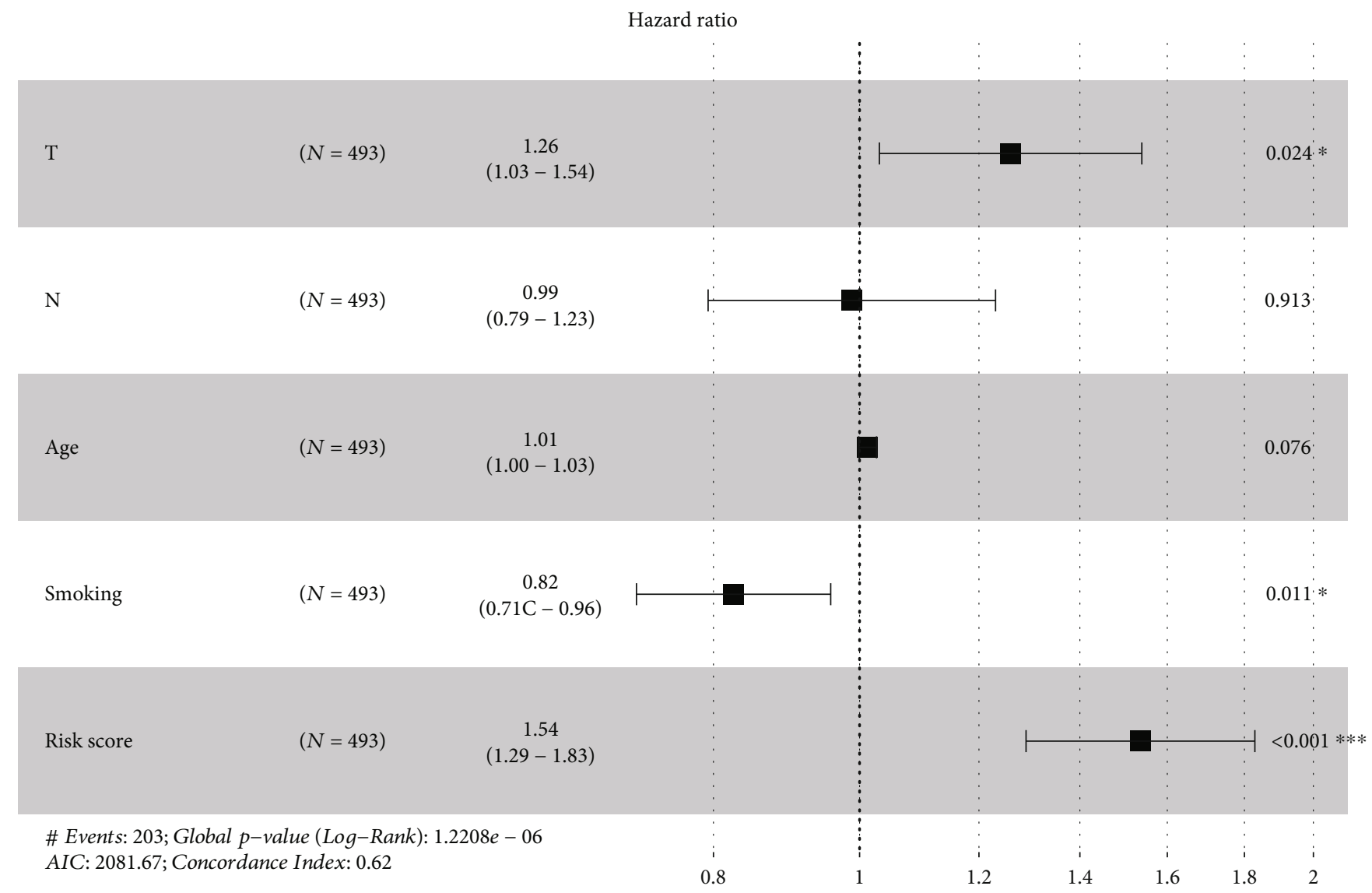

FIGURE 8: The forest plot constructed by combining the stage-T, stage-N, age, smoking, and risk score.

3.4. Association between Risk Score Value, Signal Pathways, and Sample Clinical Features. The KEGG functional enrichment scores of all samples analyzed using the ssGSEA function of the R software GSVA package were correlated with risk score values and resulted in the acquisition of 41 relevant KEGG pathways. Cluster analysis was performed according to enrichment scores as shown in Figure 6. The most correlated pathway was the JAK/STAT signaling pathway.

The relationship between different clinical parameters (including stage $(\mathrm{T}, \mathrm{N}$, or $\mathrm{M})$, gender, subdivision, age, and smoking habit) and risk score value was explored (Figure S4). The clinical features did not reveal a relationship with risk score value, except for age, indicating that risk score was relatively independent of the evaluated clinical characteristics.

3.5. Nomogram Prediction Model Establishment. Risk score value was used in combination with clinical features to establish the nomogram model (Figure 7) in which risk score exhibited a pronounced association, with the greatest influence on survival rate prediction. This suggested that the risk model based on 14 genes displayed favorable performance in predicting the prognosis of LUSC. The forest plot based on risk score value and clinical features (Figure 8 ) indicated a risk score HR of $1.54(p<0.001)$.

\section{Discussion}

Our study developed a novel prognostic model employing 14 immune-related genes using data from the TCGA Research Network and ImmPort database. This prognostic model successfully predicted LUSC patient prognosis.

Surgical resection offers the most effective treatment for early-stage LUSC [10]. Adjuvant chemotherapy or EGFRTKI improves the survival of stage II-III lung cancer patients after surgery $[11,12]$. Therefore, adjuvant chemotherapy has been the standard care for resected stage II-III LUSC patients albeit many patients do not benefit from this form of chemotherapy. This phenomenon may be related to tumor heterogeneity. Our prediction model accurately identified early LUSC patients at high risk of recurrence.

The association between the immune system and pathogenesis, as well as the progression of malignancies, has drawn increasing attention in recent years. Unlike the rapid development of LUAD treatment strategies, LUSC treatment options have progressed more slowly. Recently, immune checkpoint inhibitors that target programmed cell death 1 (PD-1) and its ligand (PD-L1) have shifted the paradigm in LUSC treatment. To date, several anti-PD-1/PD-L1 antibodies have been approved for patients with advanced NSCLC [13-15]. Emerging evidence indicates that PD-L1 expression could predict anti-PD-1/PD-L1 therapy response in patients 
with NSCLC [16]. Inspiringly, the latest reports have demonstrated that gene profiling has the potential to predict patient response to immune checkpoint inhibitors [17-19]. In addition, the association of risk score value with relevant signal pathways was explored with JAK/STAT revealed as the most significantly correlated pathway. A previous study indicated that the JAK/STAT signaling pathway plays an important role in immunity regulation in the tumor microenvironment [20]. Given our results, drug-induced interference with the expression of this pathway may provide a new direction for LUSC treatment.

Distribution of the 14 immune-related genes was investigated in Risk-H and Risk-L samples. Seven of the 14 genes, including PTPN11, MAVS, CXCL5, PLAU, MMP9, AKT2, and HSPA5, reportedly participate in the pathological processes of the immune microenvironment, as well as the pathogenesis, malignant transformation, and progression of LUSC, which exhibited marked correlation with patient survival and prognosis [21-26]. Our results demonstrated that bioinformatics mining using available research is highly reliable and accurate. Nonetheless, the association between LUSC and EDN2 and LTBP2 genes, which may be enriched in the endogenous ligand and LTBP gene families, has not been verified in either basic or clinical studies. EDN2 is reportedly involved in regulating malignant cancer cell proliferation and invasion, which can affect cytokinemediated signaling pathways as well as modulate the activation and chemotaxis of immunocytes [27]. At the same time, LTBP2 has been established as a prognostic marker for diverse cancer types and can control tumor cell sensitivity to immunotherapy $[28,29]$. Elucidation of the roles of END2 and LTBP2 in NSCLC is currently underway in our laboratory.

There were several limitations of the present study. First, our study was based on data from public datasets without prospective testing. Second, of the immune-related genes used in the prognostic model, the roles of seven genes in NSCLC are unclear. Their prognostic value should be validated by other cohorts. Third, whether patients received immunotherapy is uncertain; therefore, the predictive value of the prognostic model for immunotherapy could not be directly evaluated.

\section{Conclusions}

We identified new prognostic markers for LUSC that contribute to classifying patients with LUSC based on their immune molecular subtypes. Our predictive prognosis model based on immune signatures has potential clinical implications for assessing the overall survival. These findings should be validated in prospective studies.
Abbreviations
NSCLC: Non-small cell lung cancer
LUAD: Lung adenocarcinoma
LUSC: Lung squamous cell carcinoma
EGFR: Epidermal growth factor receptor
ALK: Anaplastic lymphoma kinase

ROS1: $\quad$ ROS proto-oncogene 1

TNM: Tumor-node-metastasis

OS: Overall survival

TCGA: The Cancer Genome Atlas

AUC: Area under the curve

HRs: Hazard ratios

CI: $\quad$ Confidence interval

PD-1: $\quad$ Programmed cell death 1

PD-L1: Programmed cell death ligand-1.

\section{Data Availability}

The data used to support the findings of this study are included within the article.

\section{Conflicts of Interest}

The authors declare no conflicts of interest.

\section{Authors' Contributions}

Yubo Yan and Minghui Zhang, contributed equally to this work.

\section{Acknowledgments}

This study was supported by the Natural Science Foundation of Heilongjiang Province (Grant No. JJ2019LH097, JJ2019LH040) and the Haiyan Foundation of Harbin Medical University Cancer Hospital (Grant No. JJQN2018-12)

\section{Supplementary Materials}

Figure S1: distribution of overall survival in lung squamous cell carcinoma. Figure S2: verification of the reliability of the prognosis prediction model including 14 immune-related genes for LUSC patients in test set. Figure S3: verification of the stability of the prognosis prediction model including 14 immune-related genes for all the samples. Figure S4: the relationships of different clinical factors with risk score. (Supplementary Materials)

\section{References}

[1] R. L. Siegel, K. D. Miller, and A. Jemal, "Cancer statistics, 2019," CA: a Cancer Journal for Clinicians, vol. 69, no. 1, pp. 7-34, 2018.

[2] X. C. Zhang, J. Wang, G. G. Shao et al., "Comprehensive genomic and immunological characterization of Chinese non-small cell lung cancer patients," Nature Communications, vol. 10, no. 1, p. 1772, 2019.

[3] R. S. Herbst, D. Morgensztern, and C. Boshoff, "The biology and management of non-small cell lung cancer," Nature, vol. 553, no. 7689, pp. 446-454, 2018.

[4] D. R. Camidge, R. C. Doebele, and K. M. Kerr, "Comparing and contrasting predictive biomarkers for immunotherapy and targeted therapy of NSCLC," Nature Reviews Clinical Oncology, vol. 16, no. 6, pp. 341-355, 2019.

[5] G. T. Gibney, L. M. Weiner, and M. B. Atkins, "Predictive biomarkers for checkpoint inhibitor-based immunotherapy," The Lancet Oncology, vol. 17, no. 12, pp. e542-e551, 2016. 
[6] D. S. Ettinger, D. L. Aisner, D. E. Wood et al., "NCCN guidelines insights: non-small cell lung cancer, version 5.2018," Journal of the National Comprehensive Cancer Network, vol. 16, no. 7, pp. 807-821, 2018.

[7] Z. Wang, Z. Wang, X. Niu et al., "Identification of seven-gene signature for prediction of lung squamous cell carcinoma," OncoTargets and Therapy, vol. Volume 12, pp. 5979-5988, 2019.

[8] E. Martínez-Terroba, C. Behrens, J. Agorreta et al., "5 proteinbased signature for resectable lung squamous cell carcinoma improves the prognostic performance of the TNM staging," Thorax, vol. 74, no. 4, pp. 371-379, 2019.

[9] S. Hanzelmann, R. Castelo, and J. Guinney, "GSVA: gene set variation analysis for microarray and RNA-seq data," $B M C$ Bioinformatics, vol. 14, no. 1, p. 7, 2013.

[10] R. U. Osarogiagbon, G. Veronesi, W. Fang et al., "Early-stage NSCLC: advances in thoracic oncology 2018," Journal of Thoracic Oncology, vol. 14, no. 6, pp. 968-978, 2019.

[11] R. Arriagada, A. Dunant, J. P. Pignon et al., "Long-term results of the international adjuvant lung cancer trial evaluating adjuvant Cisplatin-based chemotherapy in resected lung cancer," Journal of Clinical Oncology, vol. 28, no. 1, pp. 35-42, 2010.

[12] H. Cheng, X. J. Li, X. J. Wang et al., "A meta-analysis of adjuvant EGFR-TKIs for patients with resected non-small cell lung cancer," Lung Cancer, vol. 137, pp. 7-13, 2019.

[13] J. Brahmer, K. L. Reckamp, P. Baas et al., "Nivolumab versus docetaxel in advanced squamous-cell non-small-cell lung cancer," The New England Journal of Medicine, vol. 373, no. 2, pp. 123-135, 2015.

[14] R. S. Herbst, P. Baas, D. W. Kim et al., "Pembrolizumab versus docetaxel for previously treated, PD-L1-positive, advanced non-small-cell lung cancer (KEYNOTE-010): a randomised controlled trial," Lancet, vol. 387, no. 10027, pp. 1540-1550, 2016.

[15] A. Rittmeyer, F. Barlesi, D. Waterkamp et al., "Atezolizumab versus docetaxel in patients with previously treated nonsmall-cell lung cancer (OAK): a phase 3, open-label, multicentre randomised controlled trial," Lancet, vol. 389, no. 10066, pp. 255-265, 2017.

[16] T. S. K. Mok, Y. L. Wu, I. Kudaba et al., "Pembrolizumab versus chemotherapy for previously untreated, PD-L1-expressing, locally advanced or metastatic non-small-cell lung cancer (KEYNOTE-042): a randomised, open-label, controlled, phase 3 trial," Lancet, vol. 393, no. 10183, pp. 1819-1830, 2019.

[17] H. Rizvi, F. Sanchez-Vega, K. La et al., "Molecular determinants of response to anti-programmed cell death (PD)-1 and anti-programmed death-ligand 1 (PD-L1) blockade in patients with non-small-cell lung cancer profiled with targeted nextgeneration sequencing," Journal of Clinical Oncology, vol. 36, no. 7, pp. 633-641, 2018.

[18] L. Galluzzi, T. A. Chan, G. Kroemer, J. D. Wolchok, and A. Lopez-Soto, "The hallmarks of successful anticancer immunotherapy," Science Translational Medicine, vol. 10, no. 459, article eaat7807, 2018.

[19] R. Cristescu, R. Mogg, M. Ayers et al., "Pan-tumor genomic biomarkers for PD-1 checkpoint blockade-based immunotherapy," Science, vol. 362, no. 6411, article eaar3593, 2018.

[20] B. Groner and V. von Manstein, "Jak Stat signaling and cancer: opportunities, benefits and side effects of targeted inhibition," Molecular and Cellular Endocrinology, vol. 451, pp. 1-14, 2017.
[21] C. L. Chen, T. H. Chiang, P. C. Tseng, Y. C. Wang, and C. F. Lin, "Loss of PTEN causes SHP2 activation, making lung cancer cells unresponsive to IFN- $\gamma$," Biochemical and Biophysical Research Communications, vol. 466, no. 3, pp. 578-584, 2015.

[22] L. Wang, L. Shi, J. Gu et al., "CXCL5 regulation of proliferation and migration in human non-small cell lung cancer cells," Journal of Physiology and Biochemistry, vol. 74, no. 2, pp. 313-324, 2018.

[23] T. Watanabe, T. Miura, Y. Degawa et al., "Comparison of lung cancer cell lines representing four histopathological subtypes with gene expression profiling using quantitative real-time PCR," Cancer Cell International, vol. 10, no. 1, p. 2, 2010.

[24] W. Zhang, T. Zhang, Y. Lou et al., "Placental growth factor promotes metastases of non-small cell lung cancer through MMP9," Cellular Physiology and Biochemistry, vol. 37, no. 3, pp. 1210-1218, 2015.

[25] S. Attoub, K. Arafat, N. K. Hammadi, J. Mester, and A.M. Gaben, "Akt2 knock-down reveals its contribution to human lung cancer cell proliferation, growth, motility, invasion and endothelial cell tube formation," Scientific Reports, vol. 5, no. 1, p. 12759, 2015.

[26] X. Qiu, X. Guan, W. Liu, and Y. Zhang, "DAL-1 attenuates epithelial to mesenchymal transition and metastasis by suppressing HSPA5 expression in non-small cell lung cancer," Oncology Reports, vol. 38, no. 5, pp. 3103-3113, 2017.

[27] R. Wang, C. V. Löhr, K. Fischer et al., "Epigenetic inactivation of endothelin-2 and endothelin-3 in colon cancer," International Journal of Cancer, vol. 132, no. 5, pp. 1004-1012, 2013.

[28] Y. Huang, G. Wang, C. Zhao et al., "High expression of LTBP2 contributes to poor prognosis in colorectal cancer patients and correlates with the mesenchymal colorectal cancer subtype," Disease Markers, vol. 2019, Article ID 5231269, 9 pages, 2019.

[29] J. Wang, W. J. Liang, G. T. Min, H. P. Wang, W. Chen, and $\mathrm{N}$. Yao, "LTBP2 promotes the migration and invasion of gastric cancer cells and predicts poor outcome of patients with gastric cancer," International Journal of Oncology, vol. 52, no. 6, pp. 1886-1898, 2018. 